\title{
The excessive [3]-index of all graphs
}

\author{
David Cariolaro* \\ Department of Mathematical Sciences \\ Xi'an Jiaotong-Liverpool University \\ Suzhou, Jiangsu \\ China 215123 \\ davidcariolaro@hotmail.com \\ Hung-Lin Fu \\ Department of Applied Mathematics \\ National Chiao Tung University \\ Hsin Chu \\ Taiwan 30050 \\ hlfu@math.nctu.edu.tw
}

Submitted: May 14, 2008; Accepted: Sep 25, 2009; Published: Oct 5, 2009

Mathematics Subject Classification: 05C15, 05C70

\begin{abstract}
Let $m$ be a positive integer and let $G$ be a graph. A set $\mathcal{M}$ of matchings of $G$, all of which of size $m$, is called an $[m]$-covering of $G$ if $\bigcup_{M \in \mathcal{M}} M=E(G) . G$ is called $[m]$-coverable if it has an $[m]$-covering. An $[m]$-covering $\mathcal{M}$ such that $|\mathcal{M}|$ is minimum is called an excessive $[m]$-factorization of $G$ and the number of matchings it contains is a graph parameter called excessive $[m]$-index and denoted by $\chi_{[m]}^{\prime}(G)$ (the value of $\chi_{[m]}^{\prime}(G)$ is conventionally set to $\infty$ if $G$ is not $[m]$-coverable). It is obvious that $\chi_{[1]}^{\prime}(G)=|E(G)|$ for every graph $G$, and it is not difficult to see that $\chi_{[2]}^{\prime}(G)=\max \left\{\chi^{\prime}(G),\lceil|E(G)| / 2\rceil\right\}$ for every [2]-coverable graph $G$. However the task of determining $\chi_{[m]}^{\prime}(G)$ for arbitrary $m$ and $G$ seems to increase very rapidly in difficulty as $m$ increases, and a general formula for $m \geqslant 3$ is unknown. In this paper we determine such a formula for $m=3$, thereby determining the excessive [3]-index for all graphs.
\end{abstract}

Keywords: excessive $[\mathrm{m}]$-index, excessive $[\mathrm{m}]$-factorization, matching, edge coloring

\footnotetext{
*Corresponding author
} 


\section{Introduction}

All graphs considered in this paper will be implicitly assumed to be simple, finite, undirected and to contain at least one edge and no isolated vertices. The vertex set, edge set and maximum degree of a graph $G$ are denoted by $V(G), E(G)$ and $\Delta(G)$, respectively. If $V_{1} \subset V(G)$, we denote by $<V_{1}>$ the subgraph of $G$ induced by $V_{1}$. If $E_{1} \subset E(G)$, we denote by $\left\langle E_{1}>\right.$ the graph induced by the endpoints of the edges in $E_{1}$ (notice that this graph may contains also edges which are not in $E_{1}$ ). If $E_{1}=\{e, f\}$ consists of two edges only, we use the shorthand $\langle e, f>$ instead of $\langle\{e, f\}>$. A matching of $G$ is a set of mutually non-adjacent edges. If $k$ is a nonnegative integer, a $k$-edge colouring of a graph $G$ is a map $\varphi: E(G) \rightarrow \mathcal{C}$, where $\mathcal{C}$ is a set of cardinality $k$ (called the colour set), such that adjacent edges of $G$ are mapped into distinct colours. The minimum $k$ for which a $k$-edge colouring of $G$ exists is called the chromatic index of $G$ and denoted by $\chi^{\prime}(G)$. A $\chi^{\prime}(G)$-edge colouring (i.e. one which uses as few colours as possible) is called an optimal edge colouring. For graph theoretic terminology and notations, not explicitly defined here, we follow Lovász and Plummer [7].

Let $m$ be a positive integer and let $G$ be a graph. An $[m]$-covering of $G$ is a set $\mathcal{M}=\left\{M_{1}, M_{2}, \ldots, M_{k}\right\}$ of distinct matchings of $G$, each of size exactly $m$, such that $\bigcup_{i=1}^{k} M_{i}=E(G)$. The graph $G$ is said to be $[m]$-coverable if it admits an $[m]$-covering. Clearly $G$ is $[m]$-coverable if and only if every edge of $G$ belongs to a matching of $G$ of size $m$. As a consequence, checking whether a given graph is $[m]$-coverable reduces to checking whether, for each edge $e=u v$ of $G$, the graph $G-u-v$ has a matching of size at least $m-1$, which can be done in polynomial time [6].

We define a parameter $\chi_{[m]}^{\prime}(G)$, called excessive $[m]$-index, as follows:

$$
\chi_{[m]}^{\prime}(G)=\min \{|\mathcal{M}|: \mathcal{M} \text { is an }[m] \text {-covering of } G\},
$$

with the proviso that $\min \emptyset=\infty$. Thus, by the above remark, for every graph $G$ and positive integer $m$, we can determine in polynomial time whether $\chi_{[m]}^{\prime}(G)$ is finite. If $\mathcal{M}$ is an $[m]$-covering of minimum cardinality, we call $\mathcal{M}$ an excessive $[m]$-factorization of $G$. Excessive $[m]$-factorizations were introduced by the present authors in [2], where (inter alia) the parameter $\chi_{[m]}^{\prime}(G)$ was evaluated, for arbitrary $m$, for cycles, paths, complete graphs and complete bipartite graphs and for the Petersen graph. This concept lends itself to a number of possible applications, e.g. in scheduling theory, where one could wish to find a schedule, say, for a particular process, where the fundamental constraint is that all facilities must always run at full capacity (even if this could imply repeating certain jobs or operations already performed on some of the facilities) and, subject to this condition, one wishes to complete the process in the minimum possible time.

The concept of excessive $[\mathrm{m}]$-factorization is a generalization of the concept of excessive factorization, introduced by Bonisoli and Cariolaro [1] (an excessive factorization of a graph $G$ is a minimum set of perfect matchings of $G$ whose union is $E(G)$ and the corresponding parameter is called excessive index and denoted by $\chi_{e}^{\prime}(G)$ ). Not much is known in general about the parameter $\chi_{e}^{\prime}$, except that $\chi_{e}^{\prime}(G) \geqslant \chi^{\prime}(G)$ and that the difference between $\chi_{e}^{\prime}(G)$ and $\chi^{\prime}(G)$ can be arbitrarily large [1]. The present authors recently [4] 
determined $\chi_{e}^{\prime}(G)$ for all complete multipartite graphs, which proved to be a challenging task. They also introduced [3] the related notion of excessive near 1-factorization for graphs of odd order, where the size of the matchings is assumed to be the size of a near-perfect matching. The corresponding parameter (also denoted by $\chi_{e}^{\prime}(G)$ and called excessive index) was computed, apart from some elementary classes of graphs, for all trees [3]. It was observed by Bonisoli and Cariolaro [1] that, determining the number of perfect matchings in an excessive factorization of a graph is NP-complete, since this problem is equivalent to determining the existence of a 1 -factorization of $G$. The complexity of the computation of $\chi_{[m]}^{\prime}(G)$, for a fixed value of $m$, was until recently much less clear. However Cariolaro and Rizzi [5] have now settled this problem, to the effect that, when $m$ is fixed, the parameter $\chi_{[m]}^{\prime}(G)$ can be computed in polynomial time.

Trivially, $\chi_{[1]}^{\prime}(G)=|E(G)|$ for every graph $G$. For $m=2$ the present authors [2] established the formula

$$
\chi_{[2]}^{\prime}(G)=\max \left\{\chi^{\prime}(G),\left\lceil\frac{|E(G)|}{2}\right\rceil\right\}
$$

for all [2]-coverable graphs $G$ (so that every graph $G$ either satisfies (1) or satisfies $\left.\chi_{[2]}^{\prime}(G)=\infty\right)$. In this paper we shall continue the study of the excessive $[m]$-index, by determining the excessive [3]-index for all graphs. Let $S \subset E(G)$. We call $S$ a splitting set if no two edges in $S$ belong to the same matching of size 3 of $G$. The maximum cardinality of a splitting set will be denoted by $s(G)$, and $s(G)$ will be called the "splitting index" of $G$.

We notice the following.

Lemma 1 Let $G$ be a graph. Then

$$
\chi_{[3]}^{\prime}(G) \geqslant \max \left\{\chi^{\prime}(G),\left\lceil\frac{|E(G)|}{3}\right\rceil, s(G)\right\} .
$$

Proof. The inequality $\chi_{[m]}^{\prime}(G) \geqslant \max \left\{\chi^{\prime}(G),\left\lceil\frac{|E(G)|}{m}\right\rceil\right\}$ for arbitrary $m$ is easy to see and was proved in [2, Theorem 3]. Hence, we only need to verify that $\chi_{[3]}^{\prime}(G) \geqslant s(G)$. Let $S$ be a maximum splitting set. Then, by definition, no pair of distinct edges of $S$ belong to the same matching of size 3 of $G$. Since, in order to cover $G$ we need to cover the edges of $S$, at least $s(G)=|S|$ matchings of size 3 are needed to cover $G$. This completes the proof of the lemma.

The objective of this paper is to prove that we have equality in the statement of Lemma 1, i.e. the following theorem.

Theorem 1 Let $G$ be a [3]-coverable graph. Then

$$
\chi_{[3]}^{\prime}(G)=\max \left\{\chi^{\prime}(G),\lceil|E(G)| / 3\rceil, s(G)\right\} .
$$


Unfortunately, we could not prove directly Theorem 1 (and the search for a direct proof of Theorem 1 remains an open challenge!). We shall actually prove a stronger result, namely Theorem 2 below, for the proof of which we need a case-by-case analysis. Before we state Theorem 2, we give some further definitions.

A bad quartet of $G$ is a set $Q \subset V(G)$ such that $|Q|=4$ and each edge of $G$ is incident with at least one vertex in $Q$. Let $\mathcal{B}$ denote the set of all bad quartets of $G$. We define a graph parameter $q(G)$ as follows:

$$
q(G)= \begin{cases}\max _{Q \in \mathcal{B}}|E(<Q>)| & \text { if } \mathcal{B} \neq \emptyset \\ 0 & \text { if } \mathcal{B}=\emptyset\end{cases}
$$

In other words, $q(G)$ is the maximum number of edges joining the vertices of a bad quartet, if a bad quartet exists, or 0 otherwise. Notice that $q(G) \leqslant s(G)$ for all graphs, since any two edges joining the vertices of a bad quartet cannot be extended to a matching of size 3. Hence we have, from Lemma 1, the inequality

$$
\chi_{[3]}^{\prime}(G) \geqslant \max \left\{\chi^{\prime}(G),\left\lceil\frac{|E(G)|}{3}\right\rceil, q(G)\right\}
$$

for all graphs $\mathrm{G}$.

Theorem 2 Let $G$ be a [3]-coverable graph. Let $\mathcal{H}$ denote the family of graphs illustrated $^{1}$ in Fig. 1. Then

$$
\chi_{[3]}^{\prime}(G)= \begin{cases}\max \left\{\chi^{\prime}(G),\left\lceil\frac{|E(G)|}{3}\right\rceil, q(G)\right\}+1 & \text { if } G \in \mathcal{H} \\ \max \left\{\chi^{\prime}(G),\left\lceil\frac{|E(G)|}{3}\right\rceil, q(G)\right\} & \text { otherwise. }\end{cases}
$$

We now show that Theorem 2 implies Theorem 1.

Proposition 1 Theorem 2 implies Theorem 1.

Proof. Assume the truth of Theorem 1. Then, for any [3]-coverable graph not in $\mathcal{H}$, we have

$$
\chi_{[3]}^{\prime}(G)=\max \left\{\chi^{\prime}(G),\lceil|E(G)| / 3\rceil, q(G)\right\} \leqslant \max \left\{\chi^{\prime}(G),\lceil|E(G)| / 3\rceil, s(G)\right\},
$$

so the identity stated by Theorem 1 follows immediately from Lemma 1 . If $G \in \mathcal{H}$, then as shown in Fig. 1, the identity $\chi_{[3]}^{\prime}(G)=s(G)$ holds, which, by Lemma 1, implies the identity of Theorem 1. This completes the proof.

\footnotetext{
${ }^{1}$ When it is necessary to specify a particular excessive [3]-factorization of a graph, e.g. in a pictorial representation, we usually do so by indicating a corresponding edge colouring satisfying the conditions of Lemma 2. In such case, we use the expression "colouring" or "colourable" always referring to an edge colouring of this particular type. In Fig.1 and all the subsequent illustrations, we adopt the convention that vertices drawn as circles are meant to be distinct from all other vertices, whereas vertices drawn as squares may coincide with other vertices drawn as squares (as long as multiple edges are avoided). Edges represented by a broken line (and their degree 1 endvertices) may or may not be assumed to exist.
} 


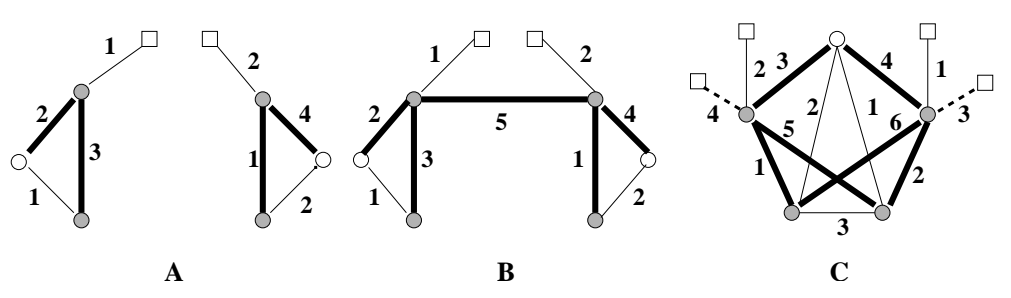

Figure 1: The family $\mathcal{H}$ referred to in the statement of Theorem 2. $\mathcal{H}$ consists of three types of graphs, labelled A,B and C, respectively. An excessive [3]-factorization of these graphs is indicated (see also the footnote to Theorem 2). The vertices of a bad quartet of $G$ containing $q(G)$ edges are shaded. The edges forming a maximum splitting set $S$ are depicted in bold. In each case we exhibit a [3]-covering of size equal to $|S|$, which proves that the [3]-covering is an excessive [3]-factorization and the splitting set is maximum by Lemma 1 . The identity stated by Theorem 2 may be verified directly for these graphs. (It always holds $\chi^{\prime}(G)=s(G)-1=\chi_{[3]}^{\prime}(G)-1$.)

We shall make use of the following lemmas. Lemma 2 was obtained independently by McDiarmid [8] and de Werra [9]. Lemmas 3 and 4 were established by the present authors in $[2]$.

Lemma 2 Let $G$ be a multigraph. If $k \geqslant \chi^{\prime}(G)$, then $G$ has a $k$-edge colouring such that every colour class $C$ satisfies $\left\lfloor\frac{|E(G)|}{k}\right\rfloor \leqslant|C| \leqslant\left\lceil\frac{|E(G)|}{k}\right\rceil$.

Lemma 3 Let $k$ be a positive integer. A graph $G$ satisfies $\chi_{[m]}^{\prime}(G) \leqslant k$ if and only if $G$ admits a $k$-edge colouring $\varphi$ such that every colour class of $\varphi$ is contained in a matching of size $m$.

Lemma 4 If a graph $G$ is $[m]$-coverable and satisfies $\frac{|E(G)|}{m} \geqslant \chi^{\prime}(G)$, then $\chi_{[m]}^{\prime}(G)=$ $\left\lceil\frac{|E(G)|}{m}\right\rceil$.

\section{Preliminary considerations}

We start by giving some additional definitions. Let $G$ be a graph. Two independent (i.e. non-adjacent) edges $e, f$ form a bad pair if they form a maximal matching, i.e. if every other edge of $G$ is adjacent to one of them. Notice that, if $\{e, f\}$ is a bad pair, then the endpoints of $e$ and $f$ form a bad quartet, as defined in Section 1.

If $\varphi$ is an edge colouring of $G$, a bad colour class is a colour class consisting of two edges of $G$ which form a bad pair, and a good colour class is a colour class which is not bad. An optimal edge colouring $\phi$ of $G$ is called a suitable colouring if every colour class of $\phi$ has size at most 3 and the number of bad colour classes is minimum.

For convenience, when we want to stress the fact that a certain edge $e$ belongs to a matching of size 3 , we say that $e$ "extends". 
In view of Lemma 1 , when proving Theorem 2 we can assume that $G$ is a graph such that

$$
\max \left\{\chi^{\prime}(G),\left\lceil\frac{|E(G)|}{3}\right\rceil\right\}<\chi_{[3]}^{\prime}(G)<\infty,
$$

so that our task is proving that $\chi_{[3]}^{\prime}(G)=q(G)$ for any graph $G$ satisfying (3), unless $G$ is one of the exceptional graphs of the family $\mathcal{H}$ (for which direct verification confirms the truth of Theorem 2). We call a graph satisfying (3) incompatible, and a graph satisfying $\chi_{[3]}^{\prime}(G)=\max \left\{\chi^{\prime}(G),\left\lceil\frac{|E(G)|}{3}\right\rceil\right\}$ compatible. We notice the following.

Lemma 5 Every incompatible graph $G$ has a suitable colouring $\phi$. Furthermore, every suitable colouring $\phi$ of $G$ has at least one bad colour class.

Proof. Let $G$ be an incompatible graph. By Lemma $4, \frac{|E(G)|}{\chi^{\prime}(G)}<3$. This implies, by Lemma 2 , the existence of an optimal edge colouring all whose colour classes have size at most 3, and hence the existence of a suitable colouring $\phi$ of $G$. Since $\phi$ is an optimal colouring, by (3) and Lemma 3 there exists a colour class of $\phi$ which is not contained in a matching of size 3 of $G$. Such colour class may not consist of a single edge, since every edge is contained in a matching of size 3 by the assumption that $\chi_{[3]}^{\prime}(G)<\infty$. Therefore such colour class consists necessarily of two edges and, since these two edges form a maximal matching, such colour class is a bad colour class. This terminates the proof.

Suitable colourings shall always be denoted by the symbol $\phi$. Now, let $G$ be an incompatible graph and let $\mathcal{S}$ denote the set of all suitable colourings of $G$. It is convenient to define an auxiliary parameter $\lambda_{*}(G)$ as follows:

$$
\lambda_{*}(G)=\min \{|E(<e, f>)|:\{e, f\} \text { is a bad colour class of } \phi, \phi \in \mathcal{S}\} .
$$

Thus $\lambda_{*}(G)$ is just the minimum number of edges in the graph induced by the quadruplets of vertices of the bad colour classes of $\phi$, where $\phi$ ranges over all suitable colourings of $G$.

In view of Lemma 5, the parameter $\lambda_{*}(G)$ is well defined for any incompatible graph $G$ and, by the definition of $\lambda_{*}(G)$ and the fact that the vertices of a bad colour class form a bad quartet, we have

$$
2 \leqslant \lambda_{*}(G) \leqslant q(G) \leqslant 6 .
$$

We now prove some further results, which will be used in the proof of Theorem 2 .

Lemma 6 There is no incompatible graph $G$ with $\Delta(G) \leqslant 2$.

Proof. Suppose there was an incompatible graph $G$ with $\Delta(G) \leqslant 2$. Then $G$ would be, in particular, [3]-coverable. If $G$ was connected, then $G$ would be a path or a cycle, but such graphs are easily seen to be compatible ([2, Proposition 8]). Hence $G$ is disconnected. Let $\phi$ be a suitable colouring of $G$ and let $\{e, f\}$ be a bad colour class. Since every edge of $G$ (other than $e$ and $f$ ) is adjacent to either $e$ or $f$, and $G$ is disconnected and has no isolated vertices, $e$ and $f$ belong to two distinct connected components of $G$. Since $e$ extends and $\{e, f\}$ is not contained in a matching of size 3 , there must be two independent edges $f^{\prime}, f^{\prime \prime}$, which are independent from $e$, and hence adjacent to $f$. Similarly, since $f$ 
extends, there must be two independent edges $e^{\prime}, e^{\prime \prime}$, adjacent to $e$. There can be no other edges in $G$, since any additional edge would have to be adjacent to either $e$ or $f$, thereby violating the condition $\Delta(G) \leqslant 2$. Thus $G$ consists of two disjoint paths of length 3, and it is easily seen that $\chi_{[3]}^{\prime}(G)=2=\chi^{\prime}(G)$, so that $G$ is compatible, contradicting the assumption.

Lemma 7 Let $e$ be an edge of an incompatible graph $G$. There exist at most two distinct edges $f, f^{\prime}$ forming a bad pair with e, respectively. Furthermore, if there exist two such edges, the subgraph of $G$ consisting of the edges not incident with $e$ has the form illustrated by Fig. 2, where $k \geqslant 1$.

Proof. Suppose $f, f^{\prime}$ are two distinct edges forming a bad pair with $e$, respectively. Let $H$ be the subgraph of $G$ consisting of the edges not incident with $e$ and their endpoints. Both $f$ and $f^{\prime}$ must be incident or coincident to every edge in $H$. In particular, $f$ and $f^{\prime}$ are adjacent. Let $x$ be their common endpoint and let $f=x y, f^{\prime}=x y^{\prime}$. Since $G$ is [3]-coverable, the edge $e$ is contained in a matching of size 3. Therefore, there exist two independent edges $g, g^{\prime}$ in $H$. Clearly $g$ and $g^{\prime}$ are distinct from $f$ and $f^{\prime}$. Since $g$ and $g^{\prime}$ are adjacent to $f$, we have, without loss of generality, $g=x x_{1}$ and $g^{\prime}=y z$. Since $g$ and $g^{\prime}$ are adjacent to $f^{\prime}$, and $y$ is not incident to $f^{\prime}, z$ must be incident to $f^{\prime}$, and, since $z \neq x$, it follows $z=y^{\prime}$. Any other edge of $H$ must be necessarily incident to both $f$ and $f^{\prime}$ and be distinct from $g^{\prime}=y y^{\prime}$, so that it must be incident with $x$. Hence $H$ has the form illustrated in Fig. 2, where $k \geqslant 1$. It is easily seen that no edge of $H$, other than $f$ and $f^{\prime}$, is incident to every other edge of $H$, and hence there can be no edge $f^{\prime \prime}$, distinct from $f$ and $f^{\prime}$, which forms a bad pair with $e$. This concludes the proof of the lemma.

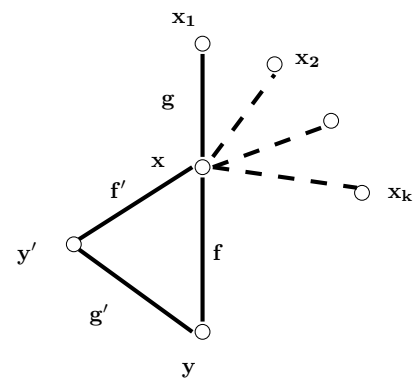

Figure 2: Structure of the subgraph of an incompatible graph $G$ containing the edges not adjacent to a fixed edge of a bad pair.

Lemma 8 Let $\phi$ be a suitable colouring of an incompatible graph $G$, and let $\{e, f\}$ be a bad colour class. Let $\delta$ be a colour not appearing on any of the edges of $\langle e, f\rangle$. Then either there exists an edge $f^{\prime} \neq f$ forming a bad pair with $e$ and coloured $\delta$, or there exists an edge $e^{\prime} \neq e$ forming a bad pair with $f$ and coloured $\delta$. 
Proof. Let $\alpha=\phi(e)=\phi(f)$. Obviously $\alpha \neq \delta$. Consider the subgraph $G(\alpha, \delta)$ of $G$ consisting of the edges coloured either $\alpha$ or $\delta$ and their endpoints. Let $C_{e}$ (respectively, $C_{f}$ ) denote the connected component of $G(\alpha, \delta)$ containing the edge $e$ (respectively, $f$ ). Clearly $C_{e} \neq C_{f}$, since $e$ and $f$ are coloured $\alpha$ and there is no edge joining $e$ and $f$ which is coloured $\delta$. It is obvious that $C_{e}$ is a path of length at most 3. Exchanging the colours $\alpha, \delta$ on $C_{e}$ we produce a colouring $\phi^{\prime}$ which is still an edge colouring of $G$ and is such that the $\alpha$-colour class is a bad colour class if and only if there exists an edge $e^{\prime} \neq e$ such that $\left\{e^{\prime}, f\right\}$ is a bad pair and $\phi^{\prime}\left(e^{\prime}\right)=\alpha$ (which is equivalent to say that $\phi\left(e^{\prime}\right)=\delta$ ). Similarly, the $\delta$-colour class of $\phi^{\prime}$ will form a bad colour class if and only if there exists an edge $f^{\prime} \neq f$ which forms a bad pair with $e$ and is such that $\phi^{\prime}\left(f^{\prime}\right)=\delta$ (or, equivalently, $\phi\left(f^{\prime}\right)=\delta$ ). If neither of the two conditions above is satisfied, $\phi^{\prime}$ has fewer colour classes forming bad pairs than $\phi$, which contradicts the assumption that $\phi$ is a suitable colouring. Hence at least one of the two conditions holds, and the lemma is proved.

Corollary 1 Let $G$ be an incompatible graph. Then $\chi^{\prime}(G) \leqslant \lambda_{*}(G)+1$.

Proof. Let $\phi$ be a suitable colouring and let $\{e, f\}$ be a bad colour class, with $\mid E(<e, f>$ ) $\mid=\lambda_{*}(G)$. Since $\phi(e)=\phi(f)$, at most $\lambda_{*}(G)-1$ distinct colours are used by $\phi$ on the edges of $\langle e, f\rangle$. By Lemma 7 and Lemma 8, there can be at most two other colours in the colour set of $\phi$. Since $\phi$ is an optimal colouring, we conclude that $\chi^{\prime}(G) \leqslant \lambda_{*}(G)+1$, as we wanted.

\section{Proof of the main theorem}

In this section we prove Theorem 2. The proof is split into several lemmas, according to the possible values of $\lambda_{*}(G)$.

Lemma 9 Let $G$ be an incompatible graph and suppose $\lambda_{*}(G)=2$. Then $G$ is isomorphic to a graph of type $A$ in Fig. 1.

Proof. Let $G$ be an incompatible graph satisfying $\lambda_{*}(G)=2$. Let $\phi$ be a suitable colouring of $G$ and let $\{e, f\}$ be a bad colour class, where $|E(<e, f>)|=2$. Let $\alpha=\phi(e)=\phi(f)$. By Lemma 6 , we have $\chi^{\prime}(G) \geqslant \Delta(G) \geqslant 3$. By Corollary 1, we have $\chi^{\prime}(G) \leqslant \lambda_{*}(G)+1=3$, so that $\chi^{\prime}(G)=3$. By Lemma 8, there exist two distinct colours $\beta$ and $\gamma$ (distinct from $\alpha$ ), an edge $e^{\prime} \neq e$, coloured $\beta$ and forming a bad pair with $f$, and an edge $f^{\prime} \neq f$, coloured $\gamma$ and forming a bad pair with $e$. Let $e=p q, e^{\prime}=p r, f=x y, f^{\prime}=x z$. Let $H_{e}\left(H_{f}\right.$, respectively) denote the subgraphs of $G$ consisting of the edges not incident with $e$ (not incident with $f$, respectively) together with their endpoints. Then $H_{e}$ and $H_{f}$ have the form prescribed by Lemma 7, where necessarily $k=1$ by the fact that $\Delta(G) \leqslant \chi^{\prime}(G)=3$. Let $x^{\prime}$ and $p^{\prime}$ denote the only vertices of degree one in $H_{e}, H_{f}$, respectively. Clearly $H_{e}$ and $H_{f}$ are edge-disjoint and every edge of $G$ is either in $H_{e}$ or in $H_{f}$. It is easily seen that $\phi\left(x x^{\prime}\right)=\beta$ and $\phi\left(p p^{\prime}\right)=\gamma$. This implies that the edge $x x^{\prime}$ may not be incident with the edge $p r$, which, together with the fact that there may not be any edge joining $e$ and $f$, implies that $x^{\prime}$ cannot belong to $H_{f}$. Similarly $p^{\prime}$ may not belong to $H_{e}$. Clearly $r$ and 
$z$ are distinct, since otherwise we have a vertex of degree 4 in $G$. Thus $H_{e}$ and $H_{f}$ are vertex-disjoint, except possibly for the vertices $x^{\prime}$ and $p^{\prime}$, which may coincide. It follows that $G$ is isomorphic to one of the graphs of type $A$ in Fig. 1, and this completes the proof.

Lemma 10 Let $G$ be an incompatible graph and suppose $\lambda_{*}(G)=3$. Then $G$ is isomorphic to a graph of type $B$ of Fig. 1.

Proof. Let $G$ be a graph satisfying the hypotheses of the lemma. Let $\phi$ be a suitable colouring having a bad colour class $\{e, f\}$, where $|E(<e, f>)|=3$. Let $E(<e, f>)=$ $\{e, f, g\}$, let $\alpha=\phi(e)=\phi(f)$, and let $\beta=\phi(g)$. By Lemma 6 , we have $\chi^{\prime}(G) \geqslant \Delta(G) \geqslant 3$. By Lemma 8, there exists a colour $\gamma \neq \alpha, \beta$ and an edge coloured $\gamma$ which forms a bad pair with either $e$ or $f$. By symmetry, we may assume this edge to be an edge $f^{\prime} \neq f$ forming a bad pair with $e$. Thus the subgraph $H_{e}$ of $G$, consisting of the edges of $G$ not incident with $e$, is of the type prescribed by Lemma 7. By Corollary $1, \chi^{\prime}(G) \leqslant \lambda_{*}(G)+1=4$. Hence $3 \leqslant \chi^{\prime}(G) \leqslant 4$.

Claim: $\chi^{\prime}(\boldsymbol{G})=4$. To prove the claim, assume $\chi^{\prime}(G)=3$. Let $f=x y, f^{\prime}=x z$, and let $x^{\prime}$ be a neighbour of $x$ of degree 1 in $H_{e}$. Since $x$ has already the three distinct neighbours $y, z, x^{\prime}$ and $\Delta(G) \leqslant \chi^{\prime}(G)=3$, the edge $g$, which joins $e$ and $f$, cannot be incident with $x$. It follows that the edge $g$ is incident with $y$. But, since $\phi(x y)=\alpha$ and $\phi(x z)=\gamma$, we necessarily have $\phi(y z)=\beta=\phi(g)$. It follows that $g$ cannot be incident to $y$ either, which yields a contradiction, proving the claim.

By the above claim and Lemma 8, there exists an edge $e^{\prime} \neq e$, forming a bad pair with $f$ and coloured $\delta$, where $\delta$ is a colour different from $\alpha, \beta, \gamma$. The graph $H_{f}$ has the form prescribed by Lemma 7. Let $f=x y, f^{\prime}=x z, e=p q, e^{\prime}=p r$. Let $p^{\prime}$ be a vertex of degree one in $H_{f}$ adjacent to $p$, and let $x^{\prime}$ be a vertex of degree one in $H_{e}$ adjacent to $x$. Notice that $r \neq z$, since otherwise the vertex $r=z$ has degree 4 , and hence it is incident to an edge coloured $\alpha$, contradicting the assumption that $e, f$ are the only edges coloured $\alpha$. We distinguish four cases, according to the pairs of endpoints of the edges $e, f$ which are matched by the edge $g$.

Case a: $\boldsymbol{g}=\boldsymbol{p} \boldsymbol{y}$. Necessarily $\phi\left(p p^{\prime}\right)=\gamma=\phi(x z)$ and hence $p^{\prime} \neq z$. Now, exchanging the colours of the edges $f$ and $f^{\prime}$, we create a colouring $\phi^{\prime}$ which is still suitable, and such that $\left\{e, f^{\prime}\right\}$ is a bad colour class. Hence, by the assumption that $\lambda_{*}(G)=3$, there must be at least one edge $h$ joining $e$ and $f^{\prime}$. Such edge cannot be incident with $p$, since $p$ has already the four neighbours $q, r, y, p^{\prime}$ (neither of which is incident with $f^{\prime}$ ) and $\Delta(G) \leqslant \chi^{\prime}(G)=4$. The edge $h$ cannot be incident with the vertex $x$, since the only edge joining $e$ and $f$ is the edge $g=p y$ by assumption. Hence $h$ must be the edge $q z$. But then $h$ is not incident with either $f$ or $e^{\prime}$, contradicting the fact that $\left\{f, e^{\prime}\right\}$ is a bad pair.

Case b: $\boldsymbol{g}=\boldsymbol{q} \boldsymbol{x}$. This case is analogous to Case 1, by symmetry.

Case c: $\boldsymbol{g}=\boldsymbol{q} \boldsymbol{y}$. As in the proof of Case a, we can claim the existence of an edge $h$ joining $e$ to $f^{\prime}$. Such edge cannot be the edge $q x$ or the edge $p x$ because only the edge $q y$ joins $e$ and $f$ in $G$, by assumption. Moreover $h \neq q z$ since $\left\{e^{\prime}, f\right\}$ is a bad pair and is not incident with $q z$. Hence $h=p z$. Similarly there is an edge $h^{\prime}$ joining $e^{\prime}$ to $f$, which must necessarily be the edge $x r$. Exchanging the colours of $e, e^{\prime}$ and (subsequently) of $f, f^{\prime}$, 
we obtain a colouring $\phi^{\prime}$ for which the $\gamma$ - and $\delta$-colour classes are good, and the $\alpha$-colour class consists of the pair $\left\{e^{\prime}, f^{\prime}\right\}$. Such pair is not a bad pair, because of the existence of the edge $g$. Thus the $\alpha$-colour class of $\phi^{\prime}$ is a good colour class, and $\phi^{\prime}$ has then less bad colour classes than $\phi$, which contradicts the assumption that $\phi$ is a suitable colouring.

Case d: $\boldsymbol{g}=\boldsymbol{p} \boldsymbol{x}$. The vertex $p$ has the four neighbours $p^{\prime}, r, q, x$, and the vertex $x$ has the four neighbours $x^{\prime}, y, z, p$. Since $\phi\left(p p^{\prime}\right)=\phi(x z)=\delta, p^{\prime}$ is distinct from $z$. Similarly, $x^{\prime}$ is distinct from $r$. Hence $H_{e}$ and $H_{f}$ are vertex disjoint, except for $p^{\prime}$ and $x^{\prime}$, which may coincide. In any case $G$ is isomorphic to one of the graphs of type B of Fig. 1, concluding the proof of the lemma.

Lemma 11 Let $G$ be an incompatible graph and suppose $\lambda_{*}(G)=4$. Then $\chi_{[3]}^{\prime}(G)=$ $q(G)$.

Proof. Let $G$ be a graph satisfying the hypotheses of the lemma. Let $\phi$ be a suitable colouring and let $\{e, f\}$ be a bad colour class, where $|E(<e, f>)|=4$. Let $E(<e, f>$ )$=\{e, f, g, h\}$ and let $e=p q, f=x y$. Let $\alpha=\phi(e)=\phi(f)$. We split the proof in two cases, according to $g, h$ being adjacent or non-adjacent.

Case 1: $\boldsymbol{g}, \boldsymbol{h}$ are adjacent. Without loss of generality, assume $g=p x, h=p y$. Let $\phi(g)=\beta, \phi(h)=\gamma$. By Corollary 1 ,

$$
4 \leqslant \Delta(G) \leqslant \chi^{\prime}(G) \leqslant \lambda_{*}(G)+1=5
$$

Hence $\chi^{\prime}(G)=4$ or 5 . Assume first $\chi^{\prime}(G)=4$. By Lemma 8, there is a colour $\delta \notin\{\alpha, \beta, \gamma\}$ and an edge coloured $\delta$ which forms a bad pair with either $e$ or $f$. Assume first that this edge is an edge $e^{\prime} \neq e$ forming a bad pair with $f$. By the structure of $H_{f}$ prescribed by Lemma 7 and the fact that $\Delta(G) \leqslant \chi^{\prime}(G)=4$, $e^{\prime}$ cannot be incident with $p$. But then $e^{\prime}$ is incident with $q$, i.e. $e^{\prime}=q r$ for some $r$, and there is, by Lemma 7 , an edge $r p$. Since there are edges adjacent to $e^{\prime}$ and coloured $\alpha, \beta, \gamma, \delta$, no such colour can be used for $e^{\prime}$, which contradicts the assumption that $\chi^{\prime}(G)=4$. Hence $e^{\prime}$ does not exist, and there is an edge $f^{\prime} \neq f$, coloured $\delta$ and forming a bad pair with $e$. By symmetry we can assume $f^{\prime}$ to be incident with $x$, say $f^{\prime}=x z$. By Lemma 7, there is an edge $z y$ and an edge $x x^{\prime}$, where $x^{\prime} \notin\{x, y, p, q, z\}$. The vertex $y$ is, by Lemma 7 , adjacent only to vertices $p, x, z$. Exchanging the colours of $f$ and $f^{\prime}$ we create a suitable colouring $\phi^{\prime}$ having $\left\{e, f^{\prime}\right\}$ as a bad colour class. Hence, using the assumption that $\lambda_{*}(G)=4$, there must be two edges joining $e$ and $f^{\prime}$ in $G$, one of which is the edge $g$. The other edge cannot be the edge $p z$, since it would have to be coloured $\delta=\phi(x z)$, which is impossible, and cannot be the edge $q x$ because of the assumption that $E(<e, f>)=\{e, f, g, h\}$. Hence such edge is necessarily the edge $q z$. To extend $g$ there must be at least one edge of the form $q q^{\prime}$, where $q^{\prime} \notin\{p, q, x, y, z\}$. Since $f$ extends, there must be one edge of the form $p p^{\prime}$, where $p^{\prime} \notin\{p, q, x, y\}$. The only possible other edge must have the form $q q^{\prime \prime}$, where $q^{\prime \prime} \notin\{p, q, x, y, z\}$. In any case the graph can be coloured as in Fig. 3.

Hence, we can now assume that $\chi^{\prime}(G)=5$. In this case there are two colours $\delta, \epsilon$, which do not appear on $E(<e, f>)$, and hence there is an edge $f^{\prime} \neq f$, forming a bad pair with $e$ and coloured $\delta$, and an edge $e^{\prime} \neq e$, forming a bad pair with $f$ and coloured 


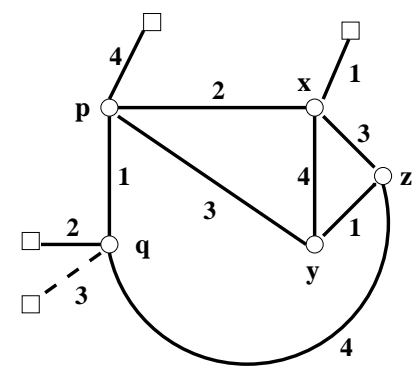

Figure 3

$\epsilon$. By symmetry, we can assume that $f^{\prime}$ has the form $f^{\prime}=x z$. We distinguish two cases (1a and $1 b)$, according to $e^{\prime}$ being incident to $p$ or to $q$.

Case 1a: $\boldsymbol{e}^{\prime}=\boldsymbol{q r}$. Necessarily $r \neq z$, since otherwise the edge $r p$ would be incident to some edges coloured $\alpha, \beta, \gamma, \delta, \epsilon$, and hence could not be allocated a colour. Exchanging the colours of $e$ and $e^{\prime}$, we create a suitable colouring $\phi^{\prime}$ having $\left\{e^{\prime}, f\right\}$ as a bad colour class. Hence, using the assumption that $\lambda_{*}(G)=4$, there must be two edges joining $e^{\prime}$ and $f$ in $G$. Arguing as before, we see that the these edges must necessarily be the edge $r x$ and the edge $r y$, but this contradicts the fact that, as prescribed by Lemma 7, $y$ is adjacent only to vertices $p, x, z$.

Case 1b: $\boldsymbol{e}^{\prime}=\boldsymbol{p} \boldsymbol{r}$. Assume first $r=z$. Then the vertex $q$ is adjacent only to vertices $p$ and $r$ and the vertex $y$ is adjacent only to vertices $p, x, r$. It follows that the edge $g=p x$ does not extend, contradicting the assumption. Thus $r \neq z$. Arguing as before, there are at least two edges joining the edges $e$ and $f^{\prime}$, one of which is $g$. The other edge must necessarily be the edge $p z$. But $p z$ is adjacent to some edges coloured $\alpha, \beta, \gamma, \delta$, $\epsilon$, hence cannot have any such colour, contradicting the hypothesis that $\chi^{\prime}(G)=5$. This settles Case 1 completely.

Case 2: $\boldsymbol{g}, \boldsymbol{h}$ are non-adjacent. Without loss of generality, assume $g=p x, h=q y$, where $\phi(g)=\beta$. By Lemma 6 and Corollary 1 , we have $3 \leqslant \chi^{\prime}(G) \leqslant 5$. Assume first $\chi^{\prime}(G)=3$. Then $G$ consists of the edges $e, f, g, h$ and the edges $p p^{\prime}, q q^{\prime}, x x^{\prime}, y y^{\prime}$, where $\left\{p^{\prime}, y^{\prime}\right\} \cap\left\{p, q, x, y, q^{\prime}, x^{\prime}\right\}=\emptyset$ and $\left\{q^{\prime}, x^{\prime}\right\} \cap\left\{p, q, x, y, p^{\prime}, y^{\prime}\right\}=\emptyset$ since all the edges $e, f, g, h$ extend. This graph can be easily coloured as shown in Fig. 4.

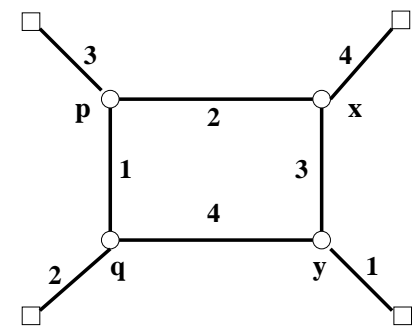

Figure 4

We now assume $\chi^{\prime}(G)=4$. In this case there is a colour $\delta$, which does not appear on 
the edges in $\langle e, f>$. By symmetry and by Lemma 8 , we can assume the existence of an edge $f^{\prime} \neq f$, coloured $\delta$, which forms a bad pair with $e$. Without loss of generality, let $f^{\prime}=x z$. Arguing as above, there must be at least two edges joining $e$ and $f^{\prime}$, one of which is $g$. The possible candidates are $p z$ and $q z$, since $q x$ cannot be an edge of $G$. Notice that $\{\phi(q y), \phi(y z)\}=\{\beta, \gamma\}$ as a consequence of our current assumptions. If $p z \in E(G), p z$ is adjacent to edges coloured $\alpha, \beta, \gamma, \delta$, and hence cannot have any such colour, contradicting the assumption that $\chi^{\prime}(G)=4$. It follows that $q z \in E(G)$. But now the configuration of the edges in $\left\langle e, f^{\prime}\right\rangle$ is exactly as in Case 1 , which is a case already settled. Therefore the case $\chi^{\prime}(G)=4$ of Case 2 can be considered settled too.

Finally, we assume $\chi^{\prime}(G)=5$. There are now exactly two colours $\delta, \epsilon$, which do not appear on $E(<e, f>)$, and an edge $f^{\prime} \neq f$ coloured $\delta$, which forms a bad pair with $e$, and an edge $e^{\prime} \neq e$, coloured $\epsilon$, which forms a bad pair with $f$. Without loss of generality, assume $\phi(e)=\phi(f)=\alpha, \phi(g)=\beta, \phi(h)=\gamma$. By symmetry, we can assume $f^{\prime}=x z$. There are now two cases to consider, according to $e^{\prime}$ being incident to $p$ or $q$, respectively. Case 2a: $\boldsymbol{e}^{\prime}=\boldsymbol{q} \boldsymbol{r}$. Assume first $r=z$. The graph $G$ contains the edges $e, f, g, h, p r, q r$, $x r, y r$ and the edges $x x^{\prime}, q q^{\prime}$ (where $\left\{x^{\prime}, q^{\prime}\right\} \cap\{p, q, r, x, y\}=\emptyset$ ), because $e, f$, extend. The only other possible edges are of the form $x x^{\prime \prime}, q q^{\prime \prime}$ (where $\left\{x^{\prime \prime}, q^{\prime \prime}\right\} \cap\{p, q, r, x, y\}=\emptyset$ ). In any case the graph is colourable as shown in Fig. 5.

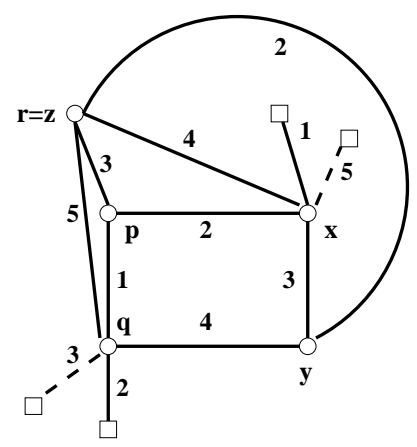

Figure 5

Hence, we may assume $r \neq z$. Arguing as above, we can claim the existence of two edges joining $e$ and $f^{\prime}$, one of which is $g$. The other edge must necessarily be the edge $q z$. Similarly, there must be two edges joining $e^{\prime}$ and $f$, one of which is $h$. This edge must necessarily be the edge $r x$. The only two possible additional edges are of the form $x x^{\prime}$ and $q q^{\prime}$, where $\left\{x^{\prime}, q^{\prime}\right\} \cap\{p, q, r, x, y, z\}=\emptyset$. In any case the graph is colourable as shown in Fig. 6.

Case 2b: $\boldsymbol{e}^{\prime}=\boldsymbol{p} \boldsymbol{r}$. Assume first $z=r$. Then the edges $q r, y r$ are adjacent to some edges coloured $\alpha, \gamma, \delta, \epsilon$, hence cannot be given a colour, which yields a contradiction. It follows that $r \neq z$. Now, arguing as above, there must be two edges joining $e$ and $f^{\prime}$, one of which is $g$. The second edge must necessarily be the edge $p z$ and is unique. But now the configuration $\left\langle e, f^{\prime}>\right.$ falls under Case 1 , hence this case can be considered settled. This concludes the proof of the lemma. 


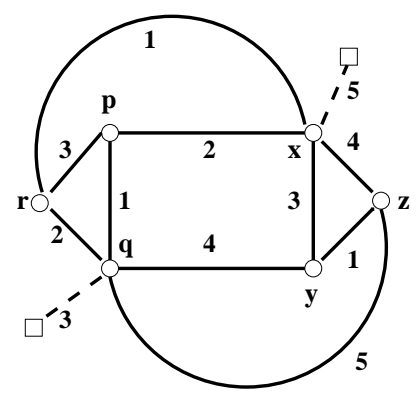

Figure 6

Lemma 12 Let $G$ be an incompatible graph and suppose $\lambda_{*}(G)=5$. Then $\chi_{[3]}^{\prime}(G)=$ $q(G)$, unless $G$ is isomorphic to one of the graphs of type $C$ in Figure 1.

Proof. Let $G$ be a graph satisfying the hypotheses of the lemma. Let $\phi$ be a suitable colouring and let $\{e, f\}$ be a bad colour class, where $|E(<e, f>)|=5$. Let $E(<e, f>$ )$=\{e, f, g, h, i\}$ and let $e=p q, f=x y, g=p x, h=p y, i=q y$. Let $\alpha=\phi(e)=\phi(f)$. By Corollary 1 and the obvious fact that $\Delta(G) \geqslant 4$, we have

$$
4 \leqslant \Delta(G) \leqslant \chi^{\prime}(G) \leqslant \lambda_{*}(G)+1=6 .
$$

Hence $\chi^{\prime}(G)=4,5$, or 6 . We split the proof into three cases, according to the value of $\chi^{\prime}(G)$.

Case 1: $\chi^{\prime}(G)=4$. In this case the graph can be easily coloured with 5 colours as shown in Fig. 7.

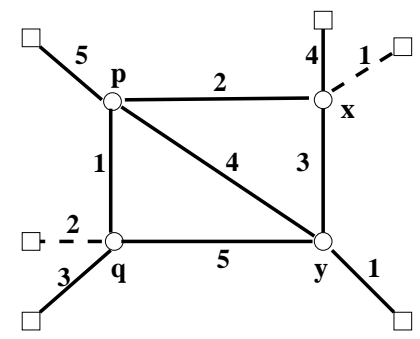

Figure 7

Case 2: $\chi^{\prime}(G)=5$. In this case there exists a colour $\delta$ which is not used on $\langle e, f\rangle$. By symmetry and Lemma 8 , we can assume the existence of an edge $f^{\prime}$ coloured $\delta$ and forming a bad pair with $e$. We distinguish two cases, according to $f^{\prime}$ being incident to $x$ or to $y$.

Case 2a: $\boldsymbol{f}^{\prime}=\boldsymbol{x} \boldsymbol{z}$. By Lemma 7, the vertex $y$ is adjacent only to vertices $p, x, z$. Exchanging the colours of $f$ and $f^{\prime}$ we create a suitable colouring such that $\left\{e, f^{\prime}\right\}$ is a bad colour class. Hence, by the assumption that $\lambda_{*}(G)=5$, there must be at least three edges joining $e$ and $f^{\prime}$ in $G$, one of which is the edge $g$. Since $q x \notin E(G)$, it follows that 
$p z, q z \in E(G)$. In addition, there must be either one or two edges of type $x x^{\prime}$ and either one or two edges of type $q q^{\prime}$, where $\left\{x^{\prime}, q^{\prime}\right\} \cap\{p, q, x, y, z\}=\emptyset$, since both $e$ and $g$ extend. There may or may not be a single edge $j$ of type $p p^{\prime}$, where $p^{\prime} \notin\{p, q, x, y, z\}$. If there is such an edge $j$, the graph is colourable with 5 colours as shown in Fig. 8. If such an edge does not exist, the graph is isomorphic to a graph of type $C$ in Fig. 1.

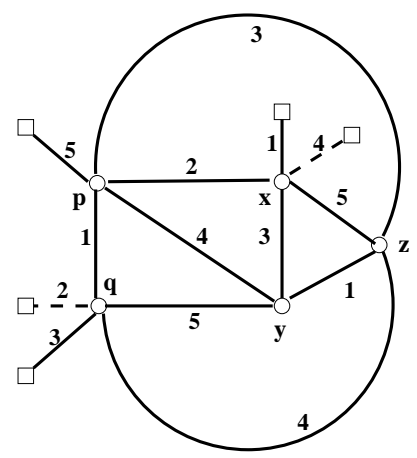

Figure 8

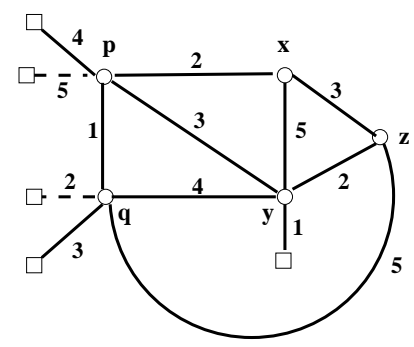

Figure 9

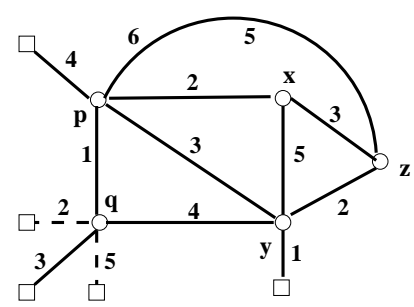

Figure 10

Case 2b: $\boldsymbol{f}^{\prime}=\boldsymbol{y} \boldsymbol{z}$. As before, we can claim the existence of at least three edges joining $e$ and $f^{\prime}$, two of which are $h, i$. A third edge could be either $q z$ or $p z$. We first assume $q z \in E(G)$. The pair $\{p y, q z\}$ is a bad pair. If $p z \notin E(G)$, we can colour with 5 colours as indicated in Fig. 9. If $p z \in E(G)$, then $q(G) \geqslant|E(<p y, q z>)|=6$, so that $q(G)=6$, 
and we can colour with 6 colours using the same colouring given in Fig. 9 and giving colour 6 to the edge $p z$. We can then assume $p z \in E(G)$ and $q z \notin E(G)$. The graph is now colourable with 5 colours as shown in Fig. 10.

Case 3: $\chi^{\prime}(G)=6$. In this case we can assume the existence of an edge $f^{\prime}$ coloured $\epsilon$ and forming a bad pair with $e$, and an edge $e^{\prime}$, coloured $\theta$, and forming a bad pair with $f$, where $\epsilon \neq \theta$ and $\epsilon, \theta \notin\{\alpha, \beta, \gamma, \delta\}$. We distinguish 4 cases, according to $e^{\prime}$ being of the form $p r$ or $q r$ and $f^{\prime}$ being of the form $x z$ or $y z$.

Case 3a: $\boldsymbol{e}^{\prime}=\boldsymbol{p} \boldsymbol{r}, \boldsymbol{f}^{\prime}=\boldsymbol{x} \boldsymbol{z}$. By Lemma 7, the vertex $q$ is adjacent to $p, r, y$ only and the vertex $y$ is adjacent to $x, p, q, z$ only. By the same argument used earlier, we can claim the existence of 3 edges joining $e$ and $f^{\prime}$, one of which is $g$. However, one of these edges must necessarily be the edge $q z$, and this is impossible unless $r=z$. But, if $r=z$, the edge $g=p x$ is not augmentable, and hence we have a contradiction.

Case 3b: $e^{\prime}=\boldsymbol{q} \boldsymbol{r}, \boldsymbol{f}^{\prime}=\boldsymbol{y} \boldsymbol{z}$. This case is symmetric to Case 3a.

Case 3c: $\boldsymbol{e}^{\prime}=\boldsymbol{q} \boldsymbol{r}, \boldsymbol{f}^{\prime}=\boldsymbol{x} \boldsymbol{z}$. Again, we can claim the existence of 3 edges joining $e$ and $f^{\prime}$, which is impossible unless $r=z$. If $r=z$, then $G$ is isomorphic to a graph of type $C$ in Fig. 1, and hence is of the desired type.

Case 3d: $\boldsymbol{e}^{\prime}=\boldsymbol{p} \boldsymbol{r}, \boldsymbol{f}^{\prime}=\boldsymbol{y} \boldsymbol{z}$. The assumption $r=z$ yields immediately a contradiction, since the edge $p y$ does not extend in that case. Hence we have $r \neq z$. Arguing as above, there must be at least 3 edges joining $e$ and $f^{\prime}$ and at least 3 edges joining $e^{\prime}$ and $f$. This implies the existence of the edges $p z$ and $r y$, respectively. The only other admissible edges are one edge of the form $p p^{\prime}$ and one edge of the form $y y^{\prime}$, where $\left\{p^{\prime}, y^{\prime}\right\} \cap\{p, q, r, x, y, z\}=$ $\emptyset$. This graph can be easily coloured with 6 colours as shown in Fig. 11. This observation concludes the proof of the lemma.

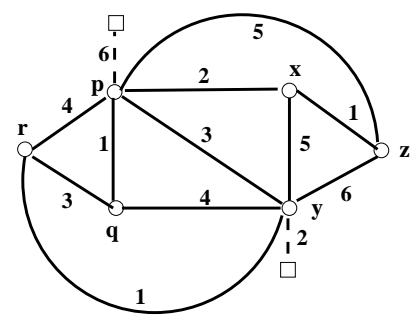

Figure 11

Lemma 13 Let $G$ be an incompatible graph and suppose $\lambda_{*}(G)=6$. Then $\chi_{[3]}^{\prime}(G)=$ $q(G)$.

Proof. Let $G$ be a graph satisfying the hypotheses of the lemma. Let $\phi$ be a suitable colouring and let $\{e, f\}$ be a bad colour class, where $|E(<e, f>)|=6$. Let $E(<$ $e, f>)=\{e, f, g, h, i, j\}$ and let $e=p q, f=x y, g=p x, h=p y, i=q y, j=q x$. Let $\alpha=\phi(e)=\phi(f)$. By Corollary 1 and the fact that the edges $e, f$ extend, we have

$$
4 \leqslant \Delta(G) \leqslant \chi^{\prime}(G) \leqslant \lambda_{*}(G)+1=7 .
$$


Hence $\chi^{\prime}(G)=4,5,6$ or 7 . We split the proof into three cases, according to the value of $\chi^{\prime}(G)$.

Case 1: $4 \leqslant \chi^{\prime}(G) \leqslant 5$. In this case $G$ is easily colourable as shown in Fig. 12 .

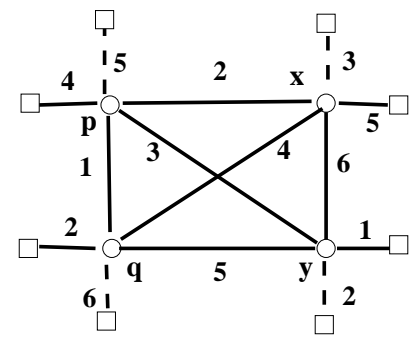

Figure 12

Case 2: $\chi^{\prime}(G)=6$. In this case there is an edge, which, by symmetry, can be assumed to be the edge $f^{\prime}=x z$ (for some $z \notin\{p, q, x, y\}$ ) such that $f^{\prime}$ is coloured $\theta$, where $\theta$ is not the colour of any edge in $\langle e, f\rangle$, and $f^{\prime}$ forms a bad pair with $e$. Arguing as above, we can claim the existence of 4 edges joining $e$ and $f^{\prime}$. Hence $p z, q z \in E(G)$. By Lemma 7, the only neighbours of $y$ are $x, p, q, z$ and there exists at least one edge of the form $x x^{\prime}$, where $x^{\prime} \notin\{p, q, x, y, z\}$. Since $j=q x$ and $g=p q$ extend, there must be at least one edge of the form $p p^{\prime}$ and one edge of the form $q q^{\prime}$, where $\left\{p^{\prime}, q^{\prime}\right\} \cap\{p, q, x, y, z\}=\emptyset$. Thus $G$ is of the type shown in Fig. 13, and can be coloured as shown.

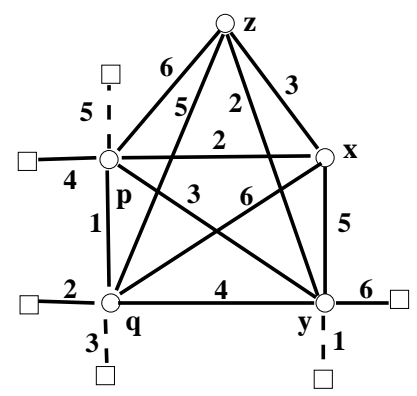

Figure 13

Case 3: $\chi^{\prime}(G)=7$. In this case there are two edges, $e^{\prime} \neq e$ and $f^{\prime} \neq f$, such that $e^{\prime}$ forms a bad pair with $f$ and $f^{\prime}$ forms a bad pair with $e$. By symmetry, we can assume $f^{\prime}=x z$ and $e^{\prime}=p r$, where $\{r, z\} \cap\{p, q, x, y\}=\emptyset$. The assumption $z=r$ yields a contradiction immediately, as the edge $g=p x$ does not extend. Hence $r \neq z$. Arguing as above, we have 4 edges joining $e$ and $f^{\prime}$ and 4 edges joining $e^{\prime}$ and $f$. Now, exchanging first the colours of $e, e^{\prime}$ and then the colours of $f, f^{\prime}$, we generate a suitable colouring $\phi^{\prime}$ such that $\alpha=\phi^{\prime}\left(e^{\prime}\right)=\phi^{\prime}\left(f^{\prime}\right)$. Necessarily the $\alpha$-colour class must be a bad colour class, otherwise $\phi^{\prime}$ has fewer bad colour classes than $\phi$, which is impossible. But this is not the case, since $\left\{e^{\prime}, f^{\prime}, q y\right\}$ is a matching of size 3. This contradiction completes the proof of the lemma. 
Proof of Theorem 2 and Theorem 1. This follows immediately by combining Lemma 9, Lemma 10, Lemma 11, Lemma 12, Lemma 13, the chain of inequalities (4) and Proposition 1.

Open problem It would be interesting to see if an assertion analogous to Theorem 1 holds for multigraphs.

\section{References}

[1] A. Bonisoli and D. Cariolaro, Excessive factorizations of regular graphs, in: A. Bondy et al. (Eds.), Graph Theory in Paris, Birkhäuser, Basel, 2007, pp. 73-84.

[2] D. Cariolaro and H.-L. Fu, Covering graphs with matchings of fixed size, to appear in Discrete Mathematics (Special Issue for the 21st British Combinatorial Conference), doi: 10.1016/j.disc.2008.09.031.

[3] D. Cariolaro and H.-L. Fu, Excessive near 1-factorizations, Discrete Mathematics, 309 (2009), 4690-4696.

[4] D. Cariolaro and H.-L. Fu, On minimum sets of 1-factors covering a complete multipartite graph, Journal of Graph Theory, 58 (2008), 239-250.

[5] D. Cariolaro and R. Rizzi, The complexity of excessive factorizations, manuscript.

[6] J. Edmonds, Paths, trees and flowers, Canad. J. Math. 17 (1965) 449-467.

[7] L. Lovász and M.D. Plummer, Matching Theory, North-Holland Mathematics Studies, Vol. 121, North-Holland, Amsterdam, 1986.

[8] C.J.H. McDiarmid, The solution of a timetabling problem, J. Inst. Math. Appl. 9 (1972) 23-34.

[9] D. de Werra, Equitable colorations of graphs, INFOR 9 (1971) 220-237. 